\title{
Influence of Nursing Students Handwashing Knowledge, Beliefs, and Attitudes on Their Handwashing Compliance
}

\author{
Omar A. Al-Khawaldeh'1, Mahmoud Al-Hussami², Muhammad Darawad² \\ ${ }^{1}$ Faculty of Nursing, Mutah University, Al-Karak, Jordan \\ ${ }^{2}$ Faculty of Nursing, The University of Jordan, Amman, Jordan \\ Email: omaralkhawaldeh175@yahoo.com, m.alhussami@ju.edu.jo, m.darawad@ju.edu.jo
}

Received 3 April 2015; accepted 29 April 2015; published 18 May 2015

Copyright ( 2015 by authors and Scientific Research Publishing Inc.

This work is licensed under the Creative Commons Attribution International License (CC BY). http://creativecommons.org/licenses/by/4.0/

(c) () Open Access

\begin{abstract}
Information regarding current status of handwashing knowledge, beliefs, attitudes and compliance with handwashing practice in nursing students in Jordan is limited. This study aims to explore handwashing knowledge, beliefs, attitudes, and self-reported compliance and to examine significant variables predicting compliance with handwashing practice among nursing students. The study used a cross-sectional survey design. Results found a statistically significant relationship between nursing students' handwashing compliance and their handwashing knowledge, attitudes, and beliefs $(r=0.68,0.65,0.58, P<0.05)$, respectively. Also, results revealed three statistically significant predictors. Namely, handwashing knowledge was the strongest predictor $(\beta=$ $0.423, P=0.000)$, followed by attitudes $(\beta=0.322, P=0.000)$, and beliefs $(\beta=0.171, P=0.027)$. Specific handwashing training courses integrated in nursing education curriculum are recommended. These courses need to consider nursing students' handwashing attitudes and beliefs in conjunction with handwashing knowledge.
\end{abstract}

\section{Keywords}

Handwashing Attitudes, Handwashing Beliefs, Handwashing Compliance, Handwashing Knowledge, Nursing Students

\section{Introduction}

Handwashing is widely recognized as an essential measure in preventing healthcare associated infections in hospitals [1]. Healthcare associated infections are one of main sources of mortality and morbidity in the hospit- 
als and contribute to the high costs of healthcare services for patients and healthcare services [2]. The Center for Disease Control and Prevention (CDC) declared that healthcare associated infections were caused by nonadherence of healthcare professionals to standard precautions of infection control and recommended handwashing practice during the course of providing patient care [3]. Although there are several evidenced-based recommendations to promote compliance with handwashing and numerous evidences of the advantages of performing handwashing, inadequate levels of compliance with handwashing among healthcare professionals continue to be reported repeatedly [4] [5].

Nurses are frequently exposed to a variety of sources of infections throughout the process of performing nursing activities [6]. Also, students' nurses are at a higher risk of contracting these infections during their clinical training courses due to their direct contacts with patients [7]. Nevertheless, it was reported that student nurses were found to have low levels of knowledge regarding infection prevention and control [8] [9], and reported low levels of handwashing compliance [10]. Thus, student nurses have an inevitable role in the spreading of healthcare associated infections [11]. The handwashing studies that have been conducted among nursing students in the Middle East region are scant, especially in Jordan [12] [13], where the focus of the studies is the healthcare professionals. As far as the authors are aware, no previous studies have been undertaken to investigate the current status of handwashing knowledge, beliefs, attitudes, and compliance among Jordanian undergraduate students' nurses. Therefore, the study specific objectives were:

1) To assess Jordanian nursing students' handwashing knowledge.

2) To assess Jordanian nursing students' handwashing compliance rate.

3) To identify Jordanian nursing students' attitudes and beliefs toward handwashing.

4) To investigate the relationships among nursing students' handwashing compliance, their demographics, knowledge, beliefs, and attitudes.

5) To identify the significant predictors of Jordanian nursing students' compliance to handwashing.

\section{Methodology}

\subsection{Design, Setting, and Sample}

A cross-sectional correlational design was utilized. The study was undertaken at nursing school in one public university in middle region of Jordan, which has more than 800 nursing students. The target population comprised all nursing students who met the inclusion criteria and agreed to participate in the study. Inclusion criteria included being a nursing student registered in years 2, 3, and 4 of the 4 year nursing bachelor program. First year nursing students were excluded as they had not entered the clinical practice in the hospitals. The sample size estimated using Cohens Tables [14]. Consequently, the estimated sample size needed to perform the regression analysis test using $\alpha=0.05$ and power $=0.80$ with six predictors was 97 subjects. However a larger sample size was targeted.

\subsection{Instrument}

The variables of the study were measured using a self-administered questionnaire that had three sections. The first section contained researcher-developed data sheet including participant's age, gender, university gradepoint average (GPA), and level of nursing education. The second section was handwashing knowledge test. The test was developed by the authors according to the world health organization hand hygiene training materials. Students were asked to answer 10 true/false questions in different aspects of handwashing. Each correct answer was allocated 1 point on a total scale score of 10 , with higher scores indicating a higher knowledge level. The content of this test was examined by a study group of three nurses who hold PhD's and several strategies were adopted to validate and refine the content of the test. These strategies included establishment the content validity through experts and pilot testing of scale on a group of nursing students who have similar characteristics as the study sample. The third section was the Handwashing Assessment Inventory (HAI) scale, which consisted of three subscales: beliefs about the handwashing outcomes, the handwashing attitudes, and self-report assessment of personal compliance with handwashing [15]. The attitude subscale contains seven items that measure different attitudes towards handwashing (e.g., convenience, necessity), while the handwashing beliefs subscale contains seven items regarding transmission of microorganisms, nurses comfort and behaviors. Both subscales utilize seven-point Likert-type format ranging from 1 (extremely unlikely) to 7 (extremely likely) with a total score 
range from 7 to 49 . Higher scores indicated positive beliefs and attitudes about the handwashing. The last subscale (self-reported compliance with handwashing) asks respondents to estimate the percentage of time (from $0 \%-100 \%$ ) they perform handwashing in nine circumstances (e.g., after procedures, after removing gloves), and the average score of the nine circumstances was utilized. In order to assess the reliability of HAI scale, Cronbach's alpha was utilized. O’Boyle et al. [15] utilized the HAI among nurses in the United States and reported a Cronbach's alpha of 0.87 for the self-reported compliance with handwashing subscale and 0.91 for the handwashing attitude subscale. This study found Cronbach's alpha to be 0.64 for the handwashing attitude subscale, 0.66 for the beliefs about handwashing outcomes subscale, and 0.80 for the self-reported compliance with handwashing subscale. The questionnaire was pilot-tested in a similar group of nursing students to ascertain the clarity and acceptability of the questionnaire and to evaluate the feasibility of the study.

\subsection{Data Collection}

The study was approved by Ethical Scientific Research Committee from the relevant university. Then, the students were requested to participate, through advertisements and flyers, in this study. The research team met the students at an assigned office, and those who showed interested were handed the questionnaire and were asked to return them to the assigned office. A cover letter was attached to the questionnaire package included explanations about purpose of the study, rights of the participants, how to complete the questionnaire and ask students for their voluntary participation. The return of completed questionnaires would indicate the consent to participate in the study. The participants' anonymity and confidentiality of data were maintained throughout the study.

\subsection{Statistical Analysis}

Descriptive statistics were used to summarize and describe participants' characteristics. Pearson correlation coefficient test was conducted to investigate the relationships between the independent variables (students' age, level of nursing education, GPA, knowledge of, beliefs about, and attitude toward handwashing) and handwashing compliance as the study dependent variable. In addition, multiple linear regression tests were performed to identify the significant variables that can predict compliance with handwashing among the independent variables. The Statistical Package for the Social Sciences (SPSS Inc., Chicago, II, USA version 19) was used to perform all statistical tests, which were run at $\alpha=0.05$ significance level (2-tailed).

\section{Results}

\subsection{Characteristics of Study Participants}

A total of 168 questionnaires were distributed and 114 (68\%) were returned and included in the analysis. The mean age of the study sample was 21.37 years $(\mathrm{SD}=1.12$, range from 19 to 26$)$ and $76 \%(\mathrm{n}=87)$ were females. The students University GPA ranged from 1.75 to 3.98 out of four (mean $=2.84$, SD $=0.55$ ). Most of the participants were in the second and third level of nursing study (45.6\%, 46.5\%, respectively).

\subsection{Level of Handwashing Knowledge}

The average score of handwashing knowledge was 7.9 out of 10 . The highest correct answer (97.4\%) was observed for the item "washing hands after removing gloves," whereas the lowest correct answer (50\%) was noted when the students answered the item "elbows should be above level of hands while doing surgical handwashing" (Table 1).

\subsection{Level of Handwashing Compliance}

The average score of self-reported handwashing compliance was 78.93\% (SD = 13.41). The highest compliance rate $($ mean $=96.92 \%, \mathrm{SD}=10.05)$ was observed for the item “wash hands after direct contact with patients' fluid or instruments," whereas the lowest compliance rate (mean $=54.91 \%$, SD = 27.56) was noted for the item "wash hands when needs to stop care" (Table 2).

\subsection{Levels of Handwashing Beliefs and Attitudes}

The average score for students' handwashing attitudes was 5.03 out of 7 (SD = 1.03). The highest attitude scores 
Table 1. Percentage of correct answer regarding handwashing knowledge $(\mathrm{N}=114)$.

Knowledge item

Correct answer (\%)

While doing surgical handwashing elbows should be above level of hands

Use of gloves is an effective substitute for handwashing

63.2

Alcohol-based hand rinse should not be used prior to patient contact

66.7

Washed hands should be left wet to stay clean

75.4

If you have an accidental exposure, immediately wash exposed skin with soap and water or flush exposed mucous membranes with water and then report incident to you instructor

89.5

In a hospital, routine handwashing is the responsibility of doctors and nurses only

91.2

Administrative personnel of the floor should wash their hands routinely

93.9

I should not wash my hands before handling or preparing food

95.6

Most important procedure for prevention of infection from germs and viruses is effective handwashing

96.5

You must wash your hands after removing your gloves

97.4

Table 2. Self-reported handwashing compliance items scores $(\mathrm{N}=114)$.

\begin{tabular}{llc}
\hline Item & Mean & SD \\
\hline 1. Handwashing hands when needs to stop care & 54.91 & 27.56 \\
2. Handwashing hands before providing care & 70.26 & 26.05 \\
3. Handwashing hands between patients & 73.95 & 25.08 \\
4. Handwashing with soap before providing care & 75.53 & 25.38 \\
5. Before touching your noise, eye, or face & 81.32 & 20.88 \\
6. After providing care or touching patient's environment & 83.42 & 20.21 \\
7. After removing gloves & 85.79 & 17.59 \\
8. After finishing patient care & 88.33 & 17.18 \\
9. After contacting patients fluids or instruments & 96.93 & 10.05 \\
\hline
\end{tabular}

SD, standard deviation.

(mean $=5.58, \mathrm{SD}=1.80$; mean $=4.83, \mathrm{SD}=2.12$ ) were for the following items "Handwashing is beneficial," and "Handwashing is necessary," respectively. The lowest attitude scores (mean = 3.85, SD = 1.94; mean = 4.56, $\mathrm{SD}=2.12$ ) were for the following items "Handwashing is soothing," and "Handwashing is reassuring," respectively (Table 3). The average score for beliefs about outcomes of handwashing was 5.54 out of 7 (SD = 0.85). The highest beliefs scores (mean $=6.61, \mathrm{SD}=0.92$; mean $=6.56, \mathrm{SD}=1.17$ ) were for the following items "Handwashing provide sense of satisfaction" and "Handwashing protect my family from infections," respectively. The lowest beliefs scores (mean $=2.54, \mathrm{SD}=1.81$; mean $=4.62$, $\mathrm{SD}=2.21$ ) were for the following items "Handwashing decreases ability to do tasks," and "Handwashing causes hands become dry," respectively (Table 3).

\subsection{Relationships between the Study Variables}

Results of Pearson correlation test analysis revealed no significant relationships among students' age, level of nursing education, and their GPA and handwashing compliance. Also, results revealed that there were statistically significant positive relationships between students' compliance with handwashing and their handwashing knowledge, attitudes, and beliefs $(r=0.68,0.65,0.58, \mathrm{P}<0.05)$ respectively (Table 4$)$.

\subsection{Predictors of Self-Reported Handwashing Compliance}

Multiple linear regression analysis was performed to estimate the independent effects of the following variables 
Table 3. Means and standards deviations for handwashing attitudes and handwashing beliefs about outcomes $(\mathrm{N}=114)$.

\begin{tabular}{lcc}
\hline Attitudes subscale items & Mean & SD \\
\hline Beneficial & 5.58 & 1.80 \\
Necessary & 4.83 & 2.12 \\
Practical & 4.81 & 1.94 \\
Convenient & 4.73 & 2.07 \\
Non-frustrating & 4.58 & 2.00 \\
Reassuring & 4.56 & 2.12 \\
Soothing & 3.85 & 1.94 \\
Beliefs about outcomes subscale items & & 1.94 \\
Sense of satisfaction & 6.61 & 1.92 \\
Protect family & 6.56 & 1.20 \\
Protect self & 6.55 & 1.19 \\
Fewer patient infections & 6.50 & 2.21 \\
Patient expectations & 6.36 & 1.81 \\
Hands becomes dry & 4.62 & 2.54 \\
Decreases ability to do tasks & & 0.96 \\
\hline SD
\end{tabular}

SD, standard deviation.

Table 4. Pearson correlation of self-reported handwashing compliance, age, level, GPA, knowledge, beliefs, and attitudes (N =114).

\begin{tabular}{|c|c|c|c|c|c|c|c|}
\hline Variables & 1 & 2 & 3 & 4 & 5 & 6 & 7 \\
\hline 1. Handwashing compliance & 1 & & & & & & \\
\hline 2. Age & 0.157 & 1 & & & & & \\
\hline 3. Level of nursing education & -0.006 & $0.516^{*}$ & 1 & & & & \\
\hline 4. GPA & 0.140 & -0.159 & $-0.334^{*}$ & 1 & & & \\
\hline 5. Knowledge & $0.678^{*}$ & 0.036 & -0.072 & 0.103 & 1 & & \\
\hline 6. Beliefs & $0.577^{*}$ & 0.136 & 0.126 & -0.074 & $0.533^{*}$ & 1 & \\
\hline 7. Attitudes & $0.654^{*}$ & 0.161 & -0.013 & -0.071 & $0.516^{*}$ & $0.527^{*}$ & 1 \\
\hline
\end{tabular}

${ }^{*}$ Correlation is significant at $\alpha=0.01$ (2-tailed).

on handwashing compliance. The variables included: handwashing knowledge, beliefs about handwashing, and attitude towards handwashing, age, level of nursing education, and GPA. As illustrated in Table 5, analysis revealed a model of three statistically significant predictors (handwashing knowledge, beliefs, attitudes), which estimated for $62.1 \%$ of the variance in nursing students' compliance with handwashing. Comparatively, nursing students' knowledge had the higher prediction effect $(\beta=0.423, \mathrm{P}=0.000)$, followed that of attitudes $(\beta=0.322$, $\mathrm{P}=0.000)$, and beliefs $(\beta=0.171, \mathrm{P}=0.027)$. Also, results revealed that nursing students' GPA, age and level of nursing education were not significant variables predicting compliance with handwashing.

\section{Discussion}

This study is the first study to examine handwashing knowledge, attitudes, belief, and compliance and to examine variables predicting compliance with handwashing in a sample of nursing students in a Jordanian context. 
Table 5. Multiple linear regression analysis of predictors of self-reported handwashing compliance $(\mathrm{N}=114)$.

\begin{tabular}{cccccc}
\hline Variables & B & SE & $\boldsymbol{\beta}$ & t & $\boldsymbol{p}$ value \\
Knowledge & 5.315 & 0.956 & 0.423 & 5.562 & $0.001^{*}$ \\
Beliefs & 0.386 & 0.172 & 0.171 & 2.238 & $0.027^{*}$ \\
Attitudes & 0.597 & 0.141 & 0.322 & 4.246 & $0.001^{*}$ \\
Age & 1.050 & 0.846 & 0.088 & 1.241 & 0.217 \\
Level of nursing education & -1.457 & 1.414 & -0.077 & -1.031 & 0.305 \\
GPA & -2.806 & 1.577 & -0.114 & -1.779 & 0.078
\end{tabular}

B, unstandardized coefficients; SE, standard error; t, as is in the regression analysis; $\mathrm{R}=0.788, \mathrm{R}^{2}=0.621$, Adjusted $\mathrm{R}^{2}=0.600, \mathrm{~F}=29.269$; ${ }^{*}$ Significant at $\alpha \leq 0.05$ (2-tailed).

It was interesting to note that average score of handwashing knowledge was less than optimal $79.17 \%$. However, this score is considered high as compared with 73.7\% among students nurses in Greece [16], 63.57\% in Slovakia [17], and 75\% in Singapore [18]. The study findings showed that average score of self-reported compliance with handwashing was $78.93 \%$, which compares favorably with other studies that reported $74.25 \%$ among Jordanian nurses [19], 63.8\% among Jordanian healthcare professionals [20], and 17\% among medical students in Saudi Arabia [21]. In contrast, it is still lower than the rate of compliance reported among students' nurses in Singapore 90\% [18] and in Turkey 80.2\% [11].

The average score of compliance with handwashing found in the present study among Jordanian nursing students is considered suboptimal as compared with recommended handwashing practice [3]. This may be related to the lack of proper education and training in handwashing practice in the study sample, which indicates that further attention is required to enhance nursing students' compliance with handwashing. Consistent with previous studies findings [19] [20] the current findings showed that the highest compliance scores were for the items (i.e., after direct contact with patients fluids or instruments, after finish of patient care) and the lowest compliance scores were for the items (i.e., before providing care, when needs to stop care). These finding may indicate that Jordanian nursing students showed more compliance with handwashing at circumstances threatening their health than circumstances threatening their patients. This implies that patient safety aspects should be reinforced during nursing clinical training courses. It was noteworthy that nursing students regarded handwashing as causing harmful effects for their skin, even though they did not believe handwashing damaging their hands. This needs further research to investigate the efficacy and user acceptance of handwashing products used in hospitals and clinical settings.

When evaluating the relationships of handwashing compliance with handwashing knowledge, beliefs, and attitudes, the variables were found to be significantly associated, suggesting that knowledge of, beliefs about, and attitudes toward handwashing influences compliance. Similarly Nasirudeen et al. [18] reported that knowledge of hand hygiene influences compliance. However, Vandijick et al. [22] and Ward [9] pointed out that higher level of handwashing knowledge does not necessarily equate improved handwashing practice. Nevertheless, inadequate handwashing knowledge is a factor that can have negative outcomes on handwashing behavior. Therefore, it was concluded that knowledge acquisition needs continuous handwashing reinforcement training programs to nursing students in the clinical settings [4].

In agreement with previous results reported among healthcare professionals [20], and among nurses [15] [19], the study findings indicated that handwashing attitudes and beliefs were significant variables that could be useful in relation to predicting compliance with handwashing. These findings suggested that nursing students are more probably be compliant with handwashing practice when they believe that more benefits and less risk are caused by handwashing. Therefore, enhancement of student nurses' positive handwashing attitudes and beliefs is an important aspect of the process of teaching handwashing practice. As poor levels of knowledge and practice regarding standard infection control precautions were reported among nursing students [8] [9] [11] and as nursing students can have a significant effects on healthcare associated infection rates [11], it is recommended to revise the nursing education curriculum to incorporate compulsory handwashing courses within the clinical train- 
ing courses. There are several interactive training and education courses on hand hygiene that could be used to influence beliefs, attitudes and behaviour in order to bring about the desired change in handwashing practice [23] [24]. Moreover, nursing students' performance of handwashing technique should be assessed and evaluated regularly through direct observational surveys to achieve optimal compliance with recommended handwashing practices. However, performing handwashing techniques properly and enhanced hand hygiene compliance requires the availability of a supportive handwashing environment within the clinical training settings [22]. The researchers encourage nursing educators and healthcare organizations managers to encourage nursing students to perform handwashing by providing appropriate handwashing products that are comfortable, easy to use and not damaging their skin. Finally, the findings of the current study should be carefully interpreted because the study was confined to a sample of nursing students drawn from one public university which limits the ability to generalize its results to other populations. In addition, the use of self-administered handwashing questionnaire for evaluating compliance with handwashing is another limitation. Therefore, replication of this study with larger sample and multiple settings and the use of combined approach of self-reported and observation methods for data collection are recommended for future research.

\section{Conclusion}

The study findings demonstrated that compliance with recommended handwashing practice among the nursing students was found to be suboptimal. Also, findings revealed that knowledge of, belief about, and attitudes toward handwashing were the significant variables predicting compliance with handwashing. To improve nursing students' competency in dealing with infection control issues and limit the transmission of diseases in the clinical practice settings, appropriate handwashing education courses are needed.

\section{Acknowledgements}

The authors are grateful to the contribution of nursing students who participate in the study.

\section{Conflicts of Interest}

None exist.

\section{References}

[1] Kilpatrick, C., Allegranzi, B. and Pittet, D. (2011) WHO First Global Patient Safety Challenge: Clean Care Is Safer Care. Contributing to the Training of Health-Care Workers around the Globe. International Journal of Infection Control, 7, 1-8. http://dx.doi.org/10.3396/ijic.V7i2.011.11

[2] World Health Organization (2009) WHO Guidelines on Hand Hygiene in Health Care 2009. http://whqlibdoc.who.int/publications/2009/9789241597906_eng.pdf

[3] Centers for Disease Control and Prevention (2002) Guidelines for Hand Hygiene in Health Care Settings. Morbidity and Mortality Weekly Report, 51, 1-48.

[4] Creedon, S. (2005) Healthcare Workers' Hand Decontamination Practices: Compliance with Recommended Guidelines. Journal of Advanced Nursing, 51, 208-216. http://dx.doi.org/10.1111/j.1365-2648.2005.03490.x

[5] Gould, D.J., Drey, N.S., Moralejo, D., Grimshaw, J. and Chudleigh, J. (2008) Interventions to Improve Hand Hygiene Compliance in Patient Care. Journal of Hospital Infection, 68, 193-202. http://dx.doi.org/10.1016/j.jhin.2007.11.013

[6] Kosgeroglu, N., Ayranci, U., Vardareli, E. and Dincer, S. (2004) Occupational Exposure to Hepatitis Infection among Turkish Nurses: Frequency of Needle Exposure, Sharps Injuries and Vaccination. Epidemiolology and Infection, 132, 27-33. http://dx.doi.org/10.1017/S0950268803001407

[7] Labrague, L.G., Rosales, R.A. and Tizon, M.M. (2012) Knowledge of and Compliance with Standard Precautions among Student Nurses. International Journal of Advanced Nursing Studies, 1, 84-97. http://dx.doi.org/10.14419/ijans.v1i2.132

[8] Ward, D.J. (2011) The Infection Control Education Needs of Nursing Students: An Interview Study with Students and Mentors. Nurse Education Today, 31, 819-824. http://dx.doi.org/10.1016/j.nedt.2010.12.017

[9] Ward, D.J. (2011) The Role of Education in the Prevention and Control of Infection: A Review of the Literature. Nurse Education Today, 31, 9-17. http://dx.doi.org/10.1016/j.nedt.2010.03.007

[10] Cole, M. (2009) Exploring the Hand Hygiene Competence of Student Nurses: A Case of Flawed Self Assessment. 
Nurse Education Today, 29, 380-388. http://dx.doi.org/10.1016/j.nedt.2008.10.010

[11] Celik, S. and Kocaslis, S. (2008) Hygienic Hand Washing among Nursing Students in Turkey. Applied Nursing Research, 21, 207-211. http://dx.doi.org/10.1016/j.apnr.2006.12.001

[12] Al-Hussami, M. and Darawad, M.W. (2012) Compliance of Nursing Students with Infection Prevention Precautions: Effectiveness of a Teaching Program. American Journal of Infection Control, 41, 332-336. http://dx.doi.org/10.1016/j.nedt.2012.06.009

[13] Darawad, M.W. and Al-Hussami, M. (2012) Jordanian Nursing Students' Knowledge of, Attitudes towards, and Compliance with Infection Control Precautions. Nurse Education Today, 33, 580-583. http://dx.doi.org/10.1016/j.nedt.2012.06.009

[14] Jocab, C. (1992) Quantitative Methods in Psychology: A Power Primer. Psychological Bulletin, 112, 155-159. http://dx.doi.org/10.1037/0033-2909.112.1.155

[15] O’Boyle, C.A., Henly, S.J. and Duckett, L.J. (2001) Nurses' Motivation to Wash Their Hands: A Standardized Measurement Approach. Applied Nursing Research, 14, 136-145. http://dx.doi.org/10.1053/apnr.2001.24412

[16] van de Mortel, T.F., Apostolopoulou, E. and Petrikkos, G. (2010) A Comparison of the Hand Hygiene Knowledge, Beliefs, and Practices of Greek Nursing and Medical Students. American Journal of Infection Control, 38, 75-77. http://dx.doi.org/10.1016/j.ajic.2009.05.006

[17] Kelcíkova, S., Skodova, Z. and Straka, S. (2011) Effectiveness of Hand Hygiene Education in a Basic Nursing School Curriculum. Public Health Nursing, 29, 152-159. http://dx.doi.org/10.1111/j.1525-1446.2011.00985.X

[18] Nasirudeen, A.M.A., Koh, J., Chin Lau, A., Li, W.J., Lim, L.S., and Ow, C.X. (2012) Hand Hygiene Knowledge and Practices of Nursing Students in Singapore. American Journal of Infection Control, 40, e241-e243. http://dx.doi.org/10.1016/j.ajic.2012.02.026

[19] Darawad, M.W., Al-Hussami, M., Al-Mhairat, I.I. and Al-Sutari, M. (2011) Investigating Jordanian Nurses' Handwashing Beliefs, Attitudes, and Compliance. American Journal of Infection Control, 40, 643-647. http://dx.doi.org/10.1016/j.ajic.2011.08.018

[20] Al-Hussami, M., Darawad, M.W. and Al-Mhairat, I.I. (2011) Predictors of Compliance Hand Hygiene Practice among Healthcare Professionals. Healthcare Infection, 16, 79-84. http://dx.doi.org/10.1071/HI11004

[21] al Kadi, A. and Salati, S.A. (2012) Hand Hygiene Practices among Medical Students. Interdisciplinary Perspectives on Infectious Diseases, 2012, Article ID: 679129. http://dx.doi.org/10.1155/2012/679129

[22] Vandijck, D.M., Labeau, S.O., De Somere, J., Claes, B. and Blot, S.I. (2008) Undergraduate Nursing Students’ Knowledge and Perception of Infection Prevention and Control. Journal of Hospital Infection, 68, 92-94.

http://dx.doi.org/10.1016/j.jhin.2007.10.013

[23] World Health Organization (2009) WHO Tools for Training and Education on the Importance of Hand Hygiene. http://www.who.int/gpsc/5may/tools/training education/en/

[24] Centers for Disease Control and Prevention (2014) Hand Hygiene Interactive Training Course. http://www.cdc.gov/handhygiene/training.html 